\title{
The Faraday rotation measure synthesis technique
}

\author{
George Heald \\ ASTRON, P.O. Box 2, 7990 AA Dwingeloo, The Netherlands \\ email: heald@astron.nl
}

\begin{abstract}
We discuss practical aspects of the novel Faraday Rotation Measure Synthesis technique, first described by Burn (1966), and recently extended and implemented by Brentjens \& de Bruyn (2005). The method takes advantage of the excellent spectral coverage provided by modern radio telescopes to reconstruct the intrinsic polarization properties along a line of sight, using a Fourier relationship between the observed polarization products and a function describing the intrinsic polarization (the Faraday dispersion function). An important consequence of the Fourier relationship and discrete frequency sampling is the need, in some cases, to deconvolve the sampling response from the reconstructed Faraday dispersion function. Practical aspects of the deconvolution procedure are discussed. We illustrate the use of the technique by summarizing a recent investigation carried out with the WSRT. We conclude by briefly describing the applicability to future programs which will be carried out with the next generation of radio telescopes such as LOFAR.
\end{abstract}

Keywords. Magnetic fields - polarization - methods: data analysis - techniques: polarimetric

\section{Introduction}

Polarimetric radio observations enable the study of synchrotron emission radiated by relativistic electrons as they are accelerated by magnetic fields. The plane of polarization of synchrotron radiation is perpendicular to the component of the magnetic field in the plane of the sky $\left(B_{\perp}\right)$. The polarization vector can be modified by the Faraday rotation effect. Faraday rotation is induced by thermal electrons, coincident with magnetic fields which are at least partially oriented along the line of sight (LOS) between the source and the observer. The amount of Faraday rotation is characterized by the Faraday rotation measure $(\mathrm{RM})$ :

$$
\mathrm{RM}=0.81 \int_{\text {source }}^{\text {observer }} n_{e} \vec{B} \cdot \mathrm{d} \vec{l},
$$

where the electron density $n_{e}$ is expressed in units $\mathrm{cm}^{-3}$, the magnetic field $B$ is in $\mu \mathrm{G}$, and the pathlength $l$ is in parsecs. The projection of the field along the line of sight $(\vec{B} \cdot \mathrm{d} \vec{l})$ is referred to as $B_{\|}$. Faraday rotation modifies the polarization angle via

$$
\chi(\lambda)=\chi_{0}+\mathrm{RM} \cdot \lambda^{2},
$$

where $\chi_{0}$ is the intrinsic polarization angle, and $\chi(\lambda)$ is the polarization angle observed at wavelength $\lambda$.

Traditionally, RM is determined by plotting the observed polarization angle as a function of the square of the observing wavelength, and performing a least-squares fit to the data. There are three potential problems with this approach,

- The observed polarization angle is only known modulo $\pi$ radians; thus, with measurements in only a few wavelength bands, the RM fit is often arbitrary. This is commonly referred to as the $n \pi$ ambiguity. For an illustration of the problem (and how closely spaced 
adjacent $\chi(\lambda)$ measurements can help to resolve the ambiguity), see Figure 1 of Rand \& Lyne (1994).

- Polarized emission with different RM values can be present in a single line of sight. The signal from these different regions mixes, and makes a linear fit inappropriate.

- Faint sources with high rotation measure will be undetectable in individual channels due to low signal-to-noise, and will remain undetectable even after integrating all channels due to bandwidth depolarization. Thus, no $\chi(\lambda)$ data points would be available for a traditional linear fit.

One way to deal with the $n \pi$ ambiguity is to rely on resolving smooth spatial gradients in the polarization angle at each frequency. With this assumption, the appropriate value of $n$ can be determined in each pixel, yielding the correct polarization angle at each frequency, and thus the true value of RM. This is the basis of the PACERMAN routine developed by Dolag et al. (2005).

However, routines such as PACERMAN cannot deal with the second and third problems mentioned above, because it is ultimately based on calculating a single RM in each LOS. In this contribution, we describe a novel technique introduced by Burn (1966), and recently extended and implemented by Brentjens \& de Bruyn (2005), who coined the term RM synthesis to describe it, and discuss its application in various astrophysical situations. The RM synthesis technique is summarized in $\S 2$. A related deconvolution issue is described in $\S 3$. The use of RM-synthesis is illustrated in $\S 4$ with results of a recent observational program carried out with the Westerbork Synthesis Radio Telescope (WSRT). We also briefly discuss future applications of this technique with the Low Frequency Array (LOFAR) in $\S 5$.

\section{RM synthesis: theory and practice}

\subsection{In theory ...}

As shown by Burn (1966), one can start by writing the observed complex polarization vector as $P\left(\lambda^{2}\right)=p I e^{2 i \chi}$, where $p$ is the fractional polarization. We now substitute Eqn. 1.2 for $\chi$, replacing RM with a generalized quantity $\phi$, the Faraday depth. Since the observed polarization vector originates from emission at all possible values of $\phi$,

$$
P\left(\lambda^{2}\right)=\int_{-\infty}^{+\infty} p I e^{2 i\left[\chi_{0}+\phi \lambda^{2}\right]} \mathrm{d} \phi
$$

which can be rewritten as

$$
P\left(\lambda^{2}\right)=\int_{-\infty}^{+\infty} F(\phi) e^{2 i \phi \lambda^{2}} \mathrm{~d} \phi,
$$

where $F(\phi)$, the Faraday dispersion function, describes the intrinsic polarized flux, as a function of the Faraday depth. Thus, we have a simple expression relating the intrinsic polarized radiation along the LOS, $F(\phi)$, to the observed quantities, $P\left(\lambda^{2}\right)$. The relation takes the form of a Fourier transform. The equation can be inverted to express the intrinsic polarization in terms of observable quantities:

$$
F(\phi)=\int_{-\infty}^{+\infty} P\left(\lambda^{2}\right) e^{-2 i \phi \lambda^{2}} \mathrm{~d} \lambda^{2} .
$$

However, one is confronted with a problem: namely, that we do not observe at wavelengths where $\lambda^{2}<0$. Nor do we observe at all values of $\lambda^{2}>0$. These issues are addressed by Brentjens \& de Bruyn (2005). 


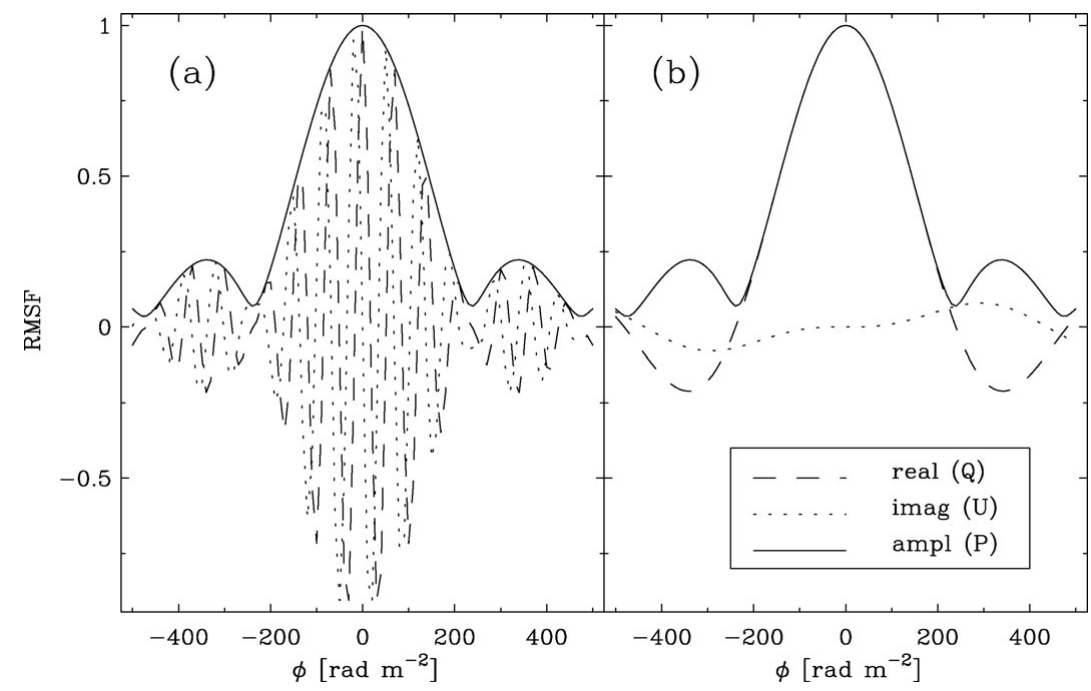

Figure 1. The RMSF of an artificial observation with $200 \mathrm{MHz}$ bandwidth, centered at 1.4 GHz, using 512 frequency channels. The RMSF is shown (a) with the factor $\lambda_{0}^{2}=0$, and (b) with $\lambda_{0}^{2}$ set to the weighted mean $\lambda^{2}$ value as advocated by Brentjens \& de Bruyn (2005). The real part of the RMSF (i.e., Stokes $Q$ ) is shown with dashed lines, the imaginary part (Stokes $U)$ with dotted lines, and the amplitude $\left(P=\sqrt{Q^{2}+U^{2}}\right)$ with solid lines.

\subsection{In practice...}

Brentjens \& de Bruyn (2005) introduce a window function, $W\left(\lambda^{2}\right)$, which is nonzero only at values of $\lambda^{2}$ which are sampled by the telescope. They proceed to show that Eqns. 2.2 and 2.3 can be rewritten to express the observed polarized emission as

$$
\tilde{P}\left(\lambda^{2}\right)=W\left(\lambda^{2}\right) P\left(\lambda^{2}\right)=W\left(\lambda^{2}\right) \int_{-\infty}^{+\infty} F(\phi) e^{2 i \phi\left(\lambda^{2}-\lambda_{0}^{2}\right)} \mathrm{d} \phi
$$

and, after some intermediate steps, the reconstructed Faraday dispersion function as

$$
\tilde{F}(\phi)=K \int_{-\infty}^{+\infty} \tilde{P}\left(\lambda^{2}\right) e^{-2 i \phi\left(\lambda^{2}-\lambda_{0}^{2}\right)} \mathrm{d} \lambda^{2}=F(\phi) \star R(\phi),
$$

where $K$ is the inverse of the integral over $W\left(\lambda^{2}\right)$, the $\star$ denotes convolution, and $R(\phi)$ is the RM spread function (RMSF) $\dagger$. The RMSF is a crucially important quantity, and is defined by

$$
R(\phi) \equiv K \int_{-\infty}^{+\infty} W\left(\lambda^{2}\right) e^{-2 i \phi\left(\lambda^{2}-\lambda_{0}^{2}\right)} \mathrm{d} \lambda^{2} .
$$

The factor $\lambda_{0}^{2}$ has been introduced in Eqns. 2.4-2.6 in order to improve the behavior of the RMSF (see Figure 1). Brentjens \& de Bruyn (2005) show that the optimal choice of $\lambda_{0}^{2}$ is the mean of the sampled $\lambda^{2}$ values, weighted by $W\left(\lambda^{2}\right)$.

Brentjens \& de Bruyn (2005) next move on to show that Eqns. 2.5 and 2.6 can be written as sums; these are the equations which define the RM synthesis technique as it

$\dagger$ Brentjens \& de Bruyn (2005) actually called this function the RM transfer function (RMTF). However, in analogy to telescope optics, the quantity $R(\phi)$ is more similar to the point spread function (PSF) than to the optical transfer function (OTF). It has therefore since been renamed the RM spread function (RMSF). 
is implemented in practice.

$$
\begin{aligned}
& \tilde{F}(\phi) \approx K \sum_{c=1}^{N} \tilde{P}_{c} e^{-2 i \phi\left(\lambda_{c}^{2}-\lambda_{0}^{2}\right)}, \\
& R(\phi) \approx K \sum_{c=1}^{N} W_{c} e^{-2 i \phi\left(\lambda_{c}^{2}-\lambda_{0}^{2}\right)},
\end{aligned}
$$

where the index $c$ refers to the individual frequency channels in which the polarized flux is observed at the radio telescope.

One of the main motivations for performing RM synthesis is to minimize the effects of the $n \pi$ ambiguity. This is best done by splitting up the observing bandwidth into many individual narrow frequency channels. By observing in this way, only the brightest polarized emission will be detected above the noise level in each narrow channel. Adding up the individual frequency channels may cause bandwidth depolarization. But the RM synthesis operation is often able to recover such low-level polarized flux. In fact, one interpretation of the RM synthesis technique is that of using a series of trial RM values, and finding the one which maximizes the signal level resulting from the coaddition of the polarized flux from all channels. In this interpretation, the flux as a function of $\phi-$ the reconstructed Faraday dispersion function - will peak at the value of $\phi$ corresponding to the actual RM of the source. At other values of $\phi$, the polarization vector rotates at the wrong rate through $\lambda^{2}$ space, the polarization vector will not constructively interfere throughout the band, and the total flux will thus be lower.

The other main reason for performing RM synthesis is to recover emission at multiple Faraday depths along a particular LOS. This circumstance can occur, for example, when a background polarized source shines through a foreground medium which both generates Faraday rotation, and also produces its own synchrotron radiation in the same volume an excellent example is the Milky Way. In such a case, polarized flux appears at different values of $\phi$, as illustrated in Figure 2.

The idealized physical situations shown in Figure 2 illustrate some of the key effects which determine the form of the Faraday dispersion function. In the top panel, a polarized background source is shown. The source has an intrinsic rotation measure of $+100 \mathrm{rad} \mathrm{m}^{-2}$. In this case, no magnetoionized medium is present along the LOS between the source and the observer, so the Faraday dispersion function is simply a delta function, with amplitude equal to the polarized flux density of the background source, and centered at $\phi=+100 \mathrm{rad} \mathrm{m}^{-2}$. In the middle panel, the situation is the same, except that a region of ionized gas $(\mathrm{A})$ is introduced along the line of sight. The region contains an ordered magnetic field inclined with respect to the LOS (but does not contain cosmic-ray electrons) in such a way that it provides an additional rotation measure of $-50 \mathrm{rad} \mathrm{m}^{-2}$. Thus, the emission from the background source is shifted to $\phi=+50 \mathrm{rad} \mathrm{m}^{-2}$. In the bottom panel, cosmic-ray electrons are added to the intervening region (B), so that in addition to the extra Faraday rotation, the region also emits its own synchrotron radiation. That which is emitted at the back of the region (furthest from the observer) is produced without a rotation measure, but accumulates a net rotation measure of $-50 \mathrm{rad} \mathrm{m}^{-2}$ by the time it propagates to the front of the region. The emission from the front of the region never accumulates any Faraday rotation on its way to the observer. The radiation emitted in the middle of the region picks up a rotation measure between those two values. In the simplest possible case, where the field distribution and particle densities are uniform, the resulting Faraday dispersion function is a tophat function, or Burn slab, as illustrated. It is present in addition to the radiation from the background source, for 


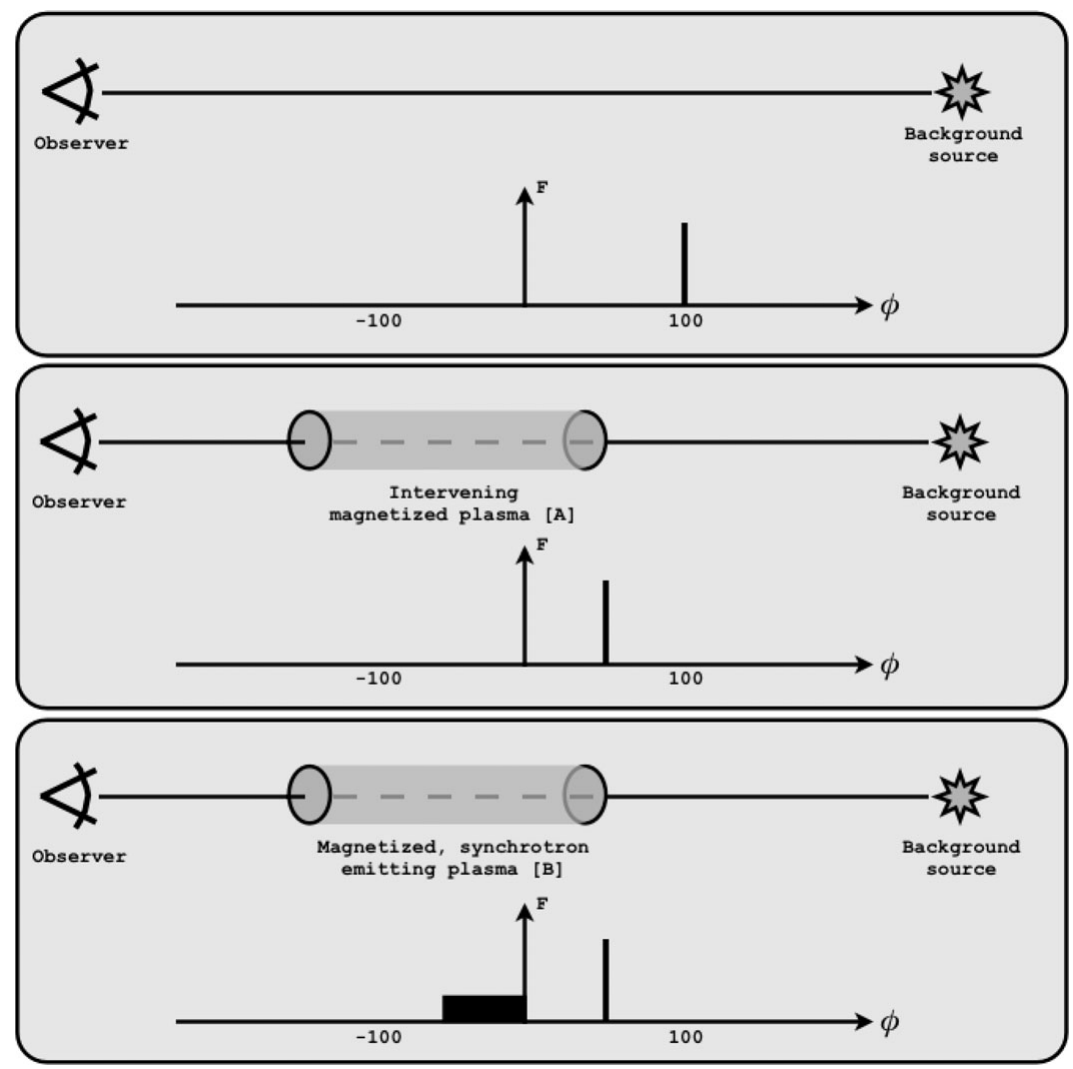

Figure 2. Three idealized physical situations along an imaginary LOS, and the Faraday dispersion functions which correspond to those situations. Refer to the text for a discussion of the individual images.

which nothing has changed relative to the picture in the middle panel (the intervening medium still changes its RM by $-50 \mathrm{rad} \mathrm{m}^{-2}$ as before).

Real objects which could make up such a picture include unresolved radio galaxies or pulsars as the background source, and the diffuse Milky Way, galaxy clusters, or galaxies as the foreground medium. Distinguishing the contributions from different objects along the LOS can be helped by including additional information about the spatial scales of, and distances to, the different objects. This is referred to as Faraday tomography.

The RM synthesis technique was originally conceived for analyzing data along a single LOS. However, many polarized sources are resolved, and it is now common to perform the inversion in Eqn. 2.7 for every spatial pixel in full Stokes $Q$ and $U$ data cubes. The output of such an operation is referred to as an "RM cube", with $\phi$ as the third axis. Frequency-dependent instrumental effects must be calibrated before generating an RM cube. For example, the synthesized beam (for an interferometer) must be the same at all frequencies. Also, the single dish primary beam attenuation must be corrected for at all frequencies. Otherwise, the frequency-dependent instrumental signal will be picked up by the RM synthesis operation, and appear in the RM cube.

\subsection{Practical issues}

There are a number of practical issues which were noted by Brentjens \& de Bruyn (2005) and which must be taken into account when doing an RM synthesis experiment. First, 
the FWHM of the RMSF determines the precision with which one can determine the RM at the peak of a Faraday dispersion function. The FWHM is inversely proportional to the full width of $\lambda^{2}$ space covered by the observations, $\Delta \lambda^{2}$. Brentjens \& de Bruyn (2005) use a proportionality constant $2 \sqrt{3}$, but Schnitzeler et al. (2008) note that empirically, a small correction is appropriate, and advocate using FWHM $=3.8 / \Delta \lambda^{2}$ instead.

As seen in $\S 2.2$, depending on the physical conditions along the line of sight, the Faraday dispersion function can contain extended structures such as Burn slabs. The signature of these structures in the observational domain is that the functional form of $\left\|P\left(\lambda^{2}\right)\right\|$ is a sinc function. Thus, at large values of $\lambda^{2}$, the degree of depolarization is high. For this reason, the sensitivity to extended Faraday structures is inversely proportional to the minimum sampled value of $\lambda^{2}$. At shorter wavelengths, the amount of depolarization due to Faraday thick emission is minimized.

One of the advantages of using the RM synthesis technique is that narrow channels can be utilized, meaning that bandwidth depolarization effects are minimized. However, they are not totally eliminated. Bandwidth depolarization becomes a concern at values of $\phi$ which cause the polarization angle to rotate by $\pi$ radians between two adjacent $\lambda^{2}$ samples. For high frequency applications, this effect only becomes important at extremely large rotation measures. But at the low frequencies sampled by LOFAR, for example, bandwidth depolarization can still be a serious concern.

A final consideration is the effect of minimizing the FWHM of the RMSF by combining observations from different frequency bands. By using, for example, a combination of a $\lambda 20 \mathrm{~cm}$ and $\lambda 6 \mathrm{~cm}$ band to increase $\Delta \lambda^{2}$, one will obtain an RMSF with a narrow FWHM, but also extremely high sidelobes. The RMSF will be dominated by a fringe characterized by the distance in $\lambda^{2}$ between the two bands, and this fringe will be damped by an envelope with width inversely proportional to the width of the individual bands. This can be thought of as similar to the interference pattern in a Young double slit experiment - the fringe spacing is set by the distance between the two slits (observing bands), and the heights of the individual fringes are determined by the width of the slits. In an observation where there is a large gap in $\lambda^{2}$ coverage, the effect is qualitatively the same.

\subsection{To weight or not to weight?}

The window function $W\left(\lambda^{2}\right)$ introduced earlier can be used to weight the individual values of $P\left(\lambda^{2}\right)$ that go into the calculation of the Faraday dispersion function. By weighting each value by the square of the signal-to-noise ratio $(S / N)$, one can hope to maximize the precision with which we determine the RM at the peak of the profile (at the expense of broadening the RMSF).

We have performed a Monte Carlo simulation to assess the effect of using such a weighting scheme. We generated mock observations of a polarized point source with a Faraday dispersion function described by $(Q=1 / \sqrt{2}, U=1 / \sqrt{2})$ at $\phi=30 \mathrm{rad} \mathrm{m}^{-2}$, and zero elsewhere. We used the same frequency coverage as was used to produce the RMSF in Figure 1. Then we added noise to the artificial observation, such that the noise level varies quadratically from channel to channel. The resulting $S / N$ ranged from 1 in the central channel, to about $1 / 12$ in the first and last channels. Then we reconstructed the Faraday dispersion function using RM synthesis, with and without $S / N$ weighting, and determined the RM at the peak of the Faraday dispersion function, $\hat{\phi}$, in each case. We ran 2500 realizations of the simulation.

The simulation results show that the weighted version of the RM synthesis calculation provides a significantly better determination of the value of $\hat{\phi}$. In Figure 3, we plot the distribution of the $\hat{\phi}$ values determined in each run of the simulation, with and without the signal-to-noise weighting. Without the weighting, the mean $\hat{\phi}$ determination was 


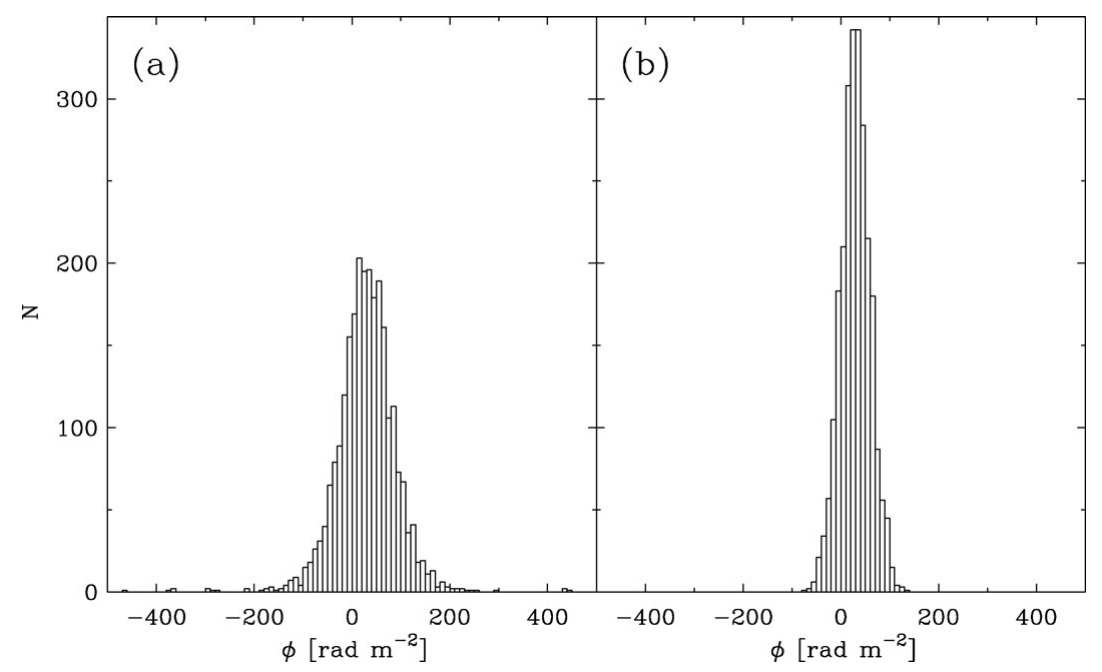

Figure 3. The distribution of $\hat{\phi}$ values determined during the Monte Carlo simulation described in the text. Results are shown from the simulated Faraday dispersion functions which were constructed (a) without weighting, and (b) with $4 \times(S / N)^{2}$ weighting. The weighting scheme serves to narrow the distribution of values. Both are centered at the actual value, $30 \mathrm{rad} \mathrm{m}^{-2}$, but the unweighted distribution is $\sim 2$ times broader.

$29.4 \pm 61.2 \mathrm{rad} \mathrm{m}^{-2}$, whereas with weighting, the determination was $29.6 \pm 30.0$, yielding a factor of 2 improvement in the precision. Note that with this frequency coverage, the expected FWHM of the RMSF is $\approx 284 \mathrm{rad} \mathrm{m}^{-2}$.

By weighting the $P\left(\lambda^{2}\right)$ values in this way, the RMSF is significantly broadened. Thus, despite the increase in precision gained, the ability to distinguish two separate features in the Faraday dispersion function will be markedly decreased. One should use different weighting schemes to optimize for the detection of different types of polarized emission, just as the weighting of visibilities measured by an aperture synthesis telescope should be optimized for the characteristics of the emission being studied.

\section{Faraday dispersion function deconvolution}

As pointed out in $\S 2.2$, after performing RM synthesis, the reconstructed Faraday dispersion function, $\tilde{F}(\phi)$, is the convolution of the actual Faraday dispersion function, $F(\phi)$, with the RMSF, $R(\phi)$. When it appears that there may be multiple features in a reconstructed Faraday dispersion function, confusion with RMSF sidelobes can make the interpretation difficult. The situation can often be improved by performing a deconvolution operation.

The algorithm is rather similar to the deconvolution routine CLEAN developed for use with aperture synthesis radio telescope images and described by Högbom (1974). The differences are (1) the deconvolution takes place in one dimension (Faraday depth) rather than two (spatial) dimensions; and (2) the functions involved are complex quantities.

The implementation of RM synthesis deconvolution proceeds as follows. First, the location of the peak of the reconstructed Faraday dispersion function, $\phi_{p}$, is searched for (either by locating the peak of $\|\tilde{F}(\phi)\|$, or alternatively the peak of cross-correlation of that function with the RMSF). Once $\phi_{p}$ is determined, the values of the real and imaginary parts of $\left\|\tilde{F}\left(\phi_{p}\right)\right\|$ are scaled by a loop gain parameter $g$, typically taken to be 0.1. This is stored as a "clean component". Next, a version of the RMSF, shifted and 


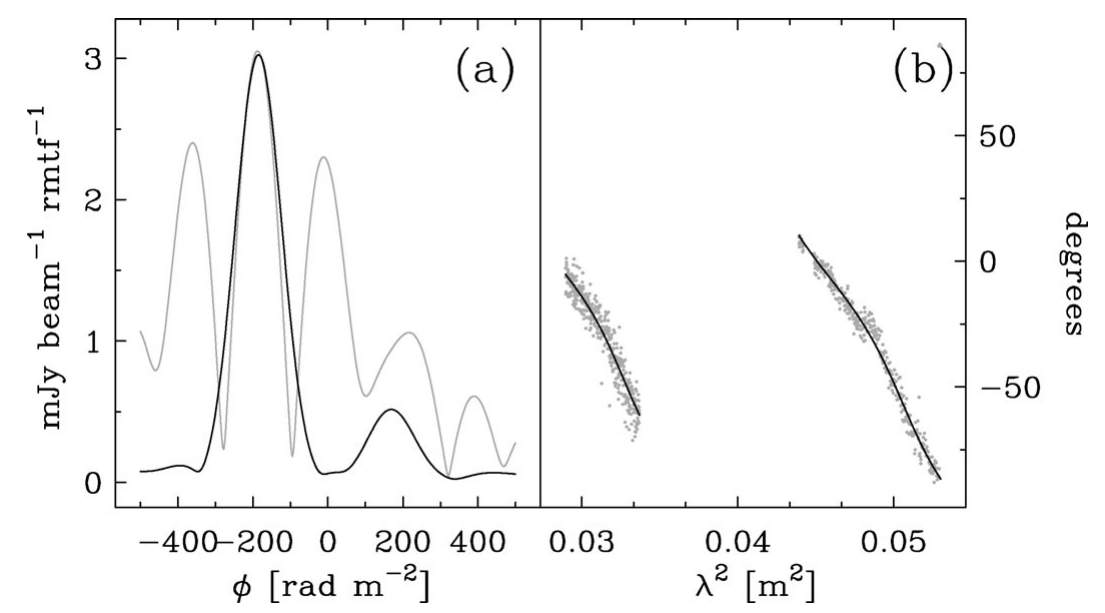

Figure 4. An example of RMCLEAN results. In panel (a) we display the Faraday dispersion function of a relatively bright polarized point source in the field of NGC 7331, observed during the WSRT-SINGS survey. The gray line is the original reconstructed Faraday dispersion function, and the black line is the deconvolved Faraday dispersion function. The resulting profile clearly shows two distinct components. In panel (b), the observed polarization angle at each frequency is plotted with gray points. The polarization angle predicted by the clean components extracted by RMCLEAN are displayed with the black line. Clearly, the model matches the data quite well. A traditional linear fit to the polarization angle vs. $\lambda^{2}$ would not have been possible in this case.

scaled to be equal to $g\left\|\tilde{F}\left(\phi_{p}\right)\right\|$ at $\phi=\phi_{p}$, is subtracted from the Faraday dispersion function. The residuals are searched for a new peak, and the loop (scale, store, subtract, and search) is repeated until the residuals are all below a user-specified threshold, or a maximum number of iterations have been performed. Finally, the clean components are convolved with a restoring function - we use a real-valued gaussian with FWHM approximately the same as the main lobe of the RMSF - and added to the residuals. The result is the deconvolved Faraday dispersion function.

This algorithm has been implemented within the MIRIAD software package, as a task called RMCLEAN, during analysis of the WSRT-SINGS survey data described in $\S 4$. 1 . The routine is available upon request.

An example of this operation is shown in Figure 4. In the left panel, the original reconstructed Faraday dispersion function, of a bright polarized point source in the WSRTSINGS survey, is compared to the deconvolved version. In the right panel, the observed $\chi\left(\lambda^{2}\right)$ values are compared to those predicted by the clean components extracted during the deconvolution operation, as a check that the behavior predicted by the model matches the data. It can also be seen that a linear fit to the $\chi\left(\lambda^{2}\right)$ data would be insufficient to characterize the situation. RM synthesis is particularly powerful in similar cases where the $S / N$ level is much lower.

\section{Applications}

In recent years, several research projects have made use of the RM synthesis technique. Many of them have been performed using data from the WSRT, which is equipped with a powerful and flexible correlator, and which can provide many channels over a large simultaneous bandwidth in all four Stokes parameters. Those projects have mostly made use of data at low frequencies, near $350 \mathrm{MHz}$; see for example the work by Brentjens (2008), Schnitzeler et al. (2008), and Bernardi et al. (2008). 


\subsection{The WSRT-SINGS survey}

One of the data sets to which RM synthesis has recently been applied is the WSRT-SINGS survey. The survey has been described by Braun et al. (2007), who also give an atlas of the observed galaxies in Stokes $I$ emission. The targets were selected mainly from the Spitzer SINGS survey (Kennicutt et al. 2003), if they have optical diameters $D_{25}>5^{\prime}$ and declination $\delta>12.5^{\circ}$. Each galaxy in the survey was observed for a total of 12 hours, split between two wide $160 \mathrm{MHz}$ frequency bands centered at $22 \mathrm{~cm}$ and $18 \mathrm{~cm}$. All four polarization products were recorded. The polarization data has been analyzed using RM synthesis (Heald et al., in prep.), which is particularly useful for this survey in order to recover faint diffuse polarized emission and its associated RM. The final noise level in Stokes $Q$ and $U$ after RM synthesis was typically $25-35 \mu \mathrm{Jy}_{\text {beam }}{ }^{-1}$ depending on the particular target. Because of the large gap between the $18 \mathrm{~cm}$ and $22 \mathrm{~cm}$ bands, the first sidelobes of the RMSF reach $\sim 78 \%$ of the main lobe. Thus, deconvolution of the Faraday dispersion functions was performed (see Figure 4 for an example which also illustrates the high sidelobes caused by the gap between the $18 \mathrm{~cm}$ and $22 \mathrm{~cm}$ bands).

RM cubes were constructed for 28 of the galaxies in the WSRT-SINGS survey. Linear polarization detections were made in 21 of 24 observed spiral galaxies, but no detections were made in the Magellanic and elliptical type galaxies. One of the fundamental products of this project is a rotation measure map for each galaxy detected in diffuse polarized emission. The RM maps were constructed by determining the value of $\phi$ at the peak of each deconvolved Faraday dispersion function. An example for NGC 6946 is shown in Figure 5. The distribution of rotation measures shows a clear gradient across the disk. A clear sinusoidal pattern is revealed by determining the average RM in wedges, and plotting as a function of the azimuthal angle in the galaxy. This sinusoidal pattern may be indicative of an axisymmetric dynamo mode, as shown in Figure 1 of Krause (1990). Using the rotation measure maps which we have determined, the observed polarization angles were corrected to their intrinsic values. This reveals the magnetic field structure in the galaxy, as shown in the last panel of Figure 5.

\section{Conclusions and future applications}

Use of the RM synthesis technique has recently become practical, thanks to the flexible correlators of modern radio telescopes such as the WSRT. The technique is very powerful in eliminating the $n \pi$ ambiguity which plagues the traditional least-squares fitting technique, and moreover provides a simple way to recover multiple polarized structures along a single LOS. In many cases, deconvolution is necessary, especially in observational programs in which large gaps in frequency coverage lead to high RMSF sidelobe levels.

Forthcoming radio telescopes, such as LOFAR (Falcke et al. 2007), which is currently being built in the Netherlands, will benefit greatly from the techniques reviewed here. At the low frequencies which will be observed $(30-240 \mathrm{MHz})$, the corresponding $\Delta \lambda^{2}$ coverage is very broad, and this leads to a very narrow RMSF response. We expect to achieve an RM precision which is more than two orders of magnitude better than in the combination of the $18 \mathrm{~cm}$ and $22 \mathrm{~cm}$ bands used in the WSRT-SINGS survey, for example. One of the LOFAR Key Science Projects (KSPs), the Magnetism KSP, will make use of the RM synthesis technique to study weak magnetic fields in the Milky Way, as well as in nearby galaxies. 

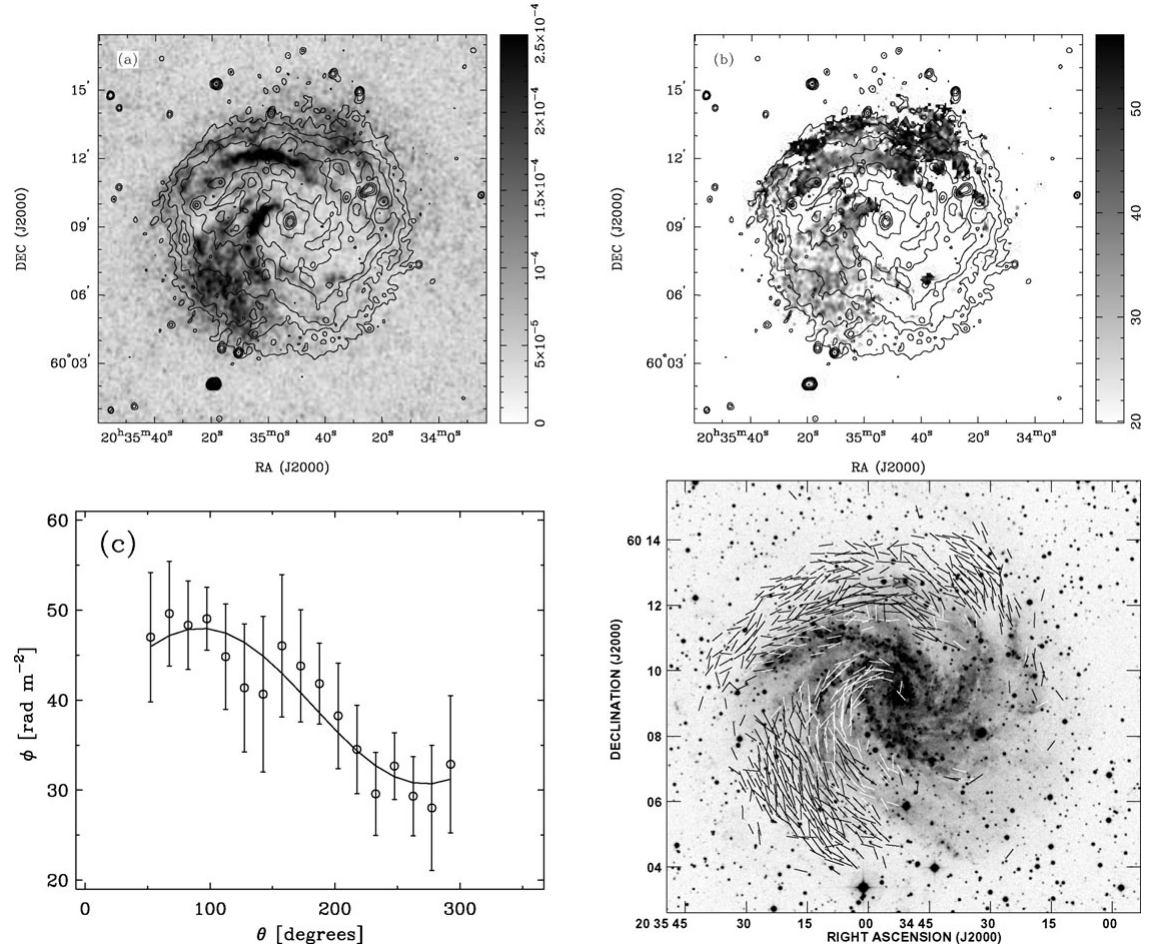

Figure 5. Linearly polarized emission in the spiral galaxy NGC 6946, observed in the WSRT-SINGS survey. (a) The distribution of the linearly polarized emission. (b) The distribution of $\phi$ values, determined as described in the text. (c) Azimuthal variation in the mean rotation measure value, together with a sinusoidal fit. (d) Magnetic field map, generated from the Faraday rotation-corrected polarization angles and overlaid on the DSS image.

\section{Acknowledgements}

The Westerbork Synthesis Radio Telescope is operated by ASTRON (Netherlands Institute for Radio Astronomy) with support from the Netherlands Foundation for Scientific Research (NWO). I would like to acknowledge my collaborators on the WSRT-SINGS work shown here, R. Braun and R. Edmonds. Many thanks to M. Brentjens for numerous enlightening conversations on these topics, and to G. de Bruyn for helpful discussions and a critical reading of this paper.

\section{References}

Bernardi, G. et al. 2008, A\&AA, submitted

Braun, R., Oosterloo, T. A., Morganti, R., Klein, U., \& Beck, R. 2007, A\&A A 461, 455

Brentjens, M. A. 2008, A\&̊ 489, 69

Burn, B. J. 1966, MNRAS 133, 67

Brentjens, M. A. \& de Bruyn, A. G. 2005, A\&SA 441, 1217

Dolag, K., Vogt, C., \& Enßlin, T. A. 2005, MNRAS 358, 726

Falcke, H. D. et al. 2007, HiA 14, 386

Högbom, J. A. 1974, A\&SAS 15, 417

Kennicutt, R. C. et al. 2003, PASP 115, 928

Krause, M. 1990, IAUS 140, 187

Rand, R. J. \& Lyne, A. G. 1994, MNRAS 268, 497

Schnitzeler, D. H. F. M., Katgert, P., \& de Bruyn, A. G. 2008, A\&SA, in press (arXiv:0810.4211) 


\section{Discussion}

KEPLEY: How does RFI affect the RM-synthesis technique?

HEALD: One should take care to remove strongly affected channels from the analysis. But as for weak RFI, aside from increasing the noise level, it should not degrade the results (as long as the RFI is not structured in the frequency domain).

Fletcher: George clearly shows (in M51) a possible $m=1$ azimuthal mode in the 18/20 cm RMs. A note of caution on the interpretation should be added (in my opinion): depolarization by unresolved magnetic fluctuations in the synchrotron emitting regions can lead to complete depolarization along part of the observed line of sight. Rotation measures at higher frequencies show a clear sign of a $m=0+2$ azimuthal pattern near the disc of M51 (Fletcher et al. in prep.), with a $m=1$ pattern (as shown by George) in a layer nearer to the observer (a thick disc or halo). Dynamo modellers should take note!

HEALD: We see a hint of a contribution from an $m=0$ mode, which may correspond to the disk component of your model, but your point about depolarization effects at these frequencies is well taken.

HAN: 1) Comment: when we do pulsar observations with 512/1024 channels inside a $256 \mathrm{MHz}$ band, PSRCHIVE software (developed by Willem van Straten) also uses RM synthesis technique for RM. Afterwards, we still check the $\triangle \mathrm{PA}$ from the upper and lower half bands for the uncertainties of the RM measurements. 2) In your observations of SINGS galaxies, your RM image is very impressive. Do you see different RM layers during the process? I think maybe there are different layers in each part inside a galaxy? You did average on that? How did you compose the final RM map?

HEALD: 1) When signal-to-noise permits, one should always check for consistency in the measurement domain. However, the real power of the RM-synthesis technique derives from the capability to extract Faraday dispersion functions from data with signal levels below the noise in each channel. 2) As Andrew Fletcher pointed out, depolarization at these frequencies likely prevents us from detecting polarization from deeply within the galaxies. But by using this technique at higher frequencies, where Faraday effects are less severe, one could probe the different layers of disk and halo. 


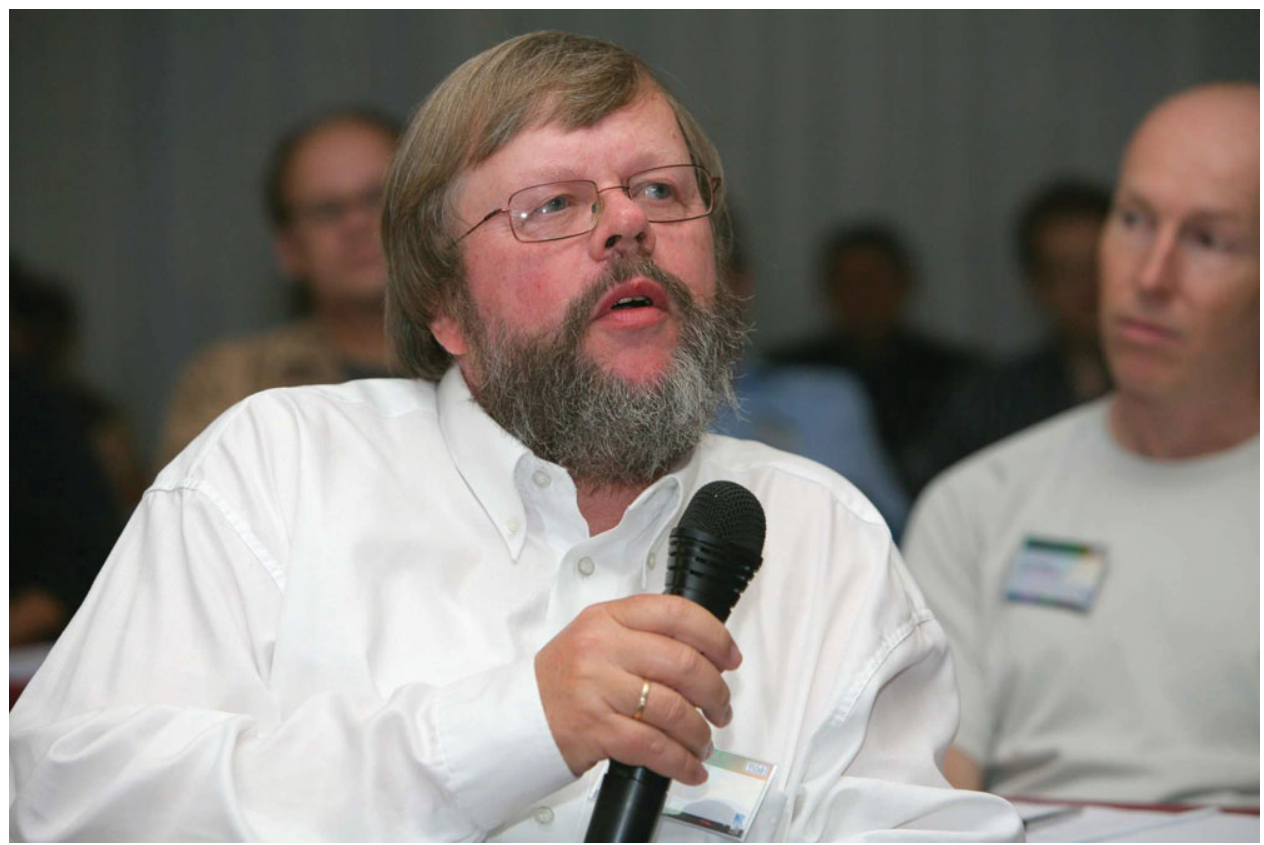

Wolfgang Reich

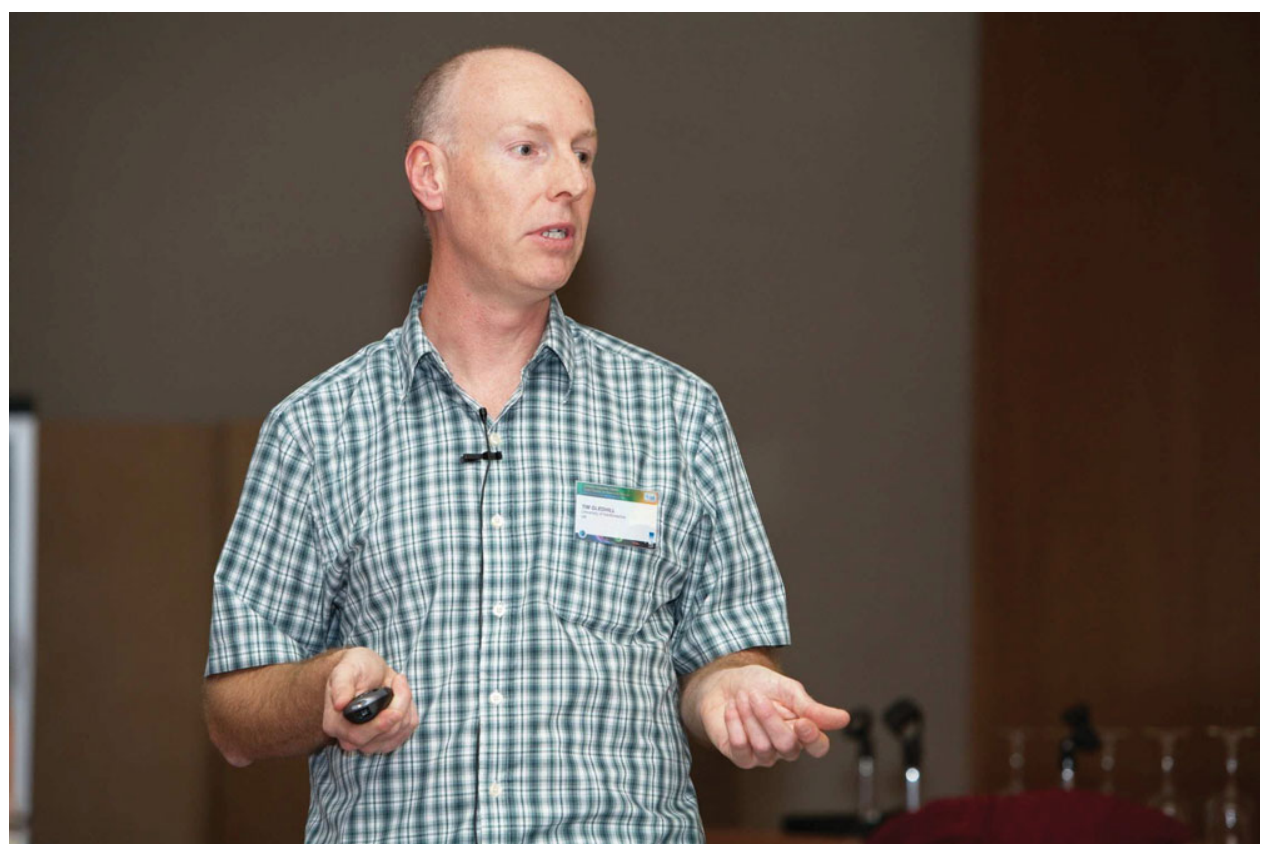

Tim Gledhill 